\title{
Reference point and confirming points; two important guides in treatment of panfacial Fx.: 3 case reports
}

\author{
Mehrnoush Momeni Roochi ${ }^{1}$, Narges Hajiani ${ }^{1}$, and Sayna Nezaminia ${ }^{1}$ \\ ${ }^{1}$ Tehran University of Medical Sciences
}

June 8, 2020

\begin{abstract}
We present 3 cases of Panfacial fractures through which we try to emphasize two important sequencing rules: "Reference point" (the most intact area to determine the starting point for fixation) and the "Confirming points" ( the points which should be checked out to confirm the exact position \& orientation).

Reference point and confirming points; two important guides in treatment of panfacial Fx.: 3 case reports

Abstract

We present 3 cases of Panfacial fractures through which we try to emphasize two important sequencing rules: "Reference point" (the most intact area to determine the starting point for fixation) and the "Confirming points" ( the points which should be checked out to confirm the exact position \& orientation).
\end{abstract}

Key words: skull fracture, fracture fixation, internal

Key clinical message

It's important to achieve and stablish a correct occlusal relationship parallel with repositioning of other fracture segments in facial skeleton, resulting rehabilitation of facial esthetic in treatment of Panfacial fractures using correct sequences

Introduction

Panfacial fractures are defined as multiple facial bone fractures including the upper, middle, and lower thirds of the face(1), which in most cases are associated with loss of soft- and hard tissue and some sequela such as blindness. (2).

Even in early management, the exact reduction and repositioning of fracture segments to their pre-traumatic position is very complicated and challenging especially in comminuted one (3).

In this matter, various sequences of reduction are offered:" Bottom-to-top", "Top-to-bottom", "Inside-out", and "Outside-inside" are the most common concepts of sequence in management of panfacial fractures in which the elected sequence is based on factors such as the pattern of fracture and the experience of surgeon (4).

The most widely used concepts are "Bottom-to-top \& Outside-to-inside"; i.e After mandibular reconstruction as a strong buttress, and stablishing the maxilla over mandible, other facial compartments including zygomatico-orbital complex will be reconstructed(4).

In this article, we presented 3 patients with emphasis on two concepts including "Reference point" and "Confirming points" that act as a guide to reduce the facial contour and improves surgical procedure. 
Reference point is defined as the intact point around the fracture area from which the reduction will start and ascertain the sequence of the reduction and fixation. For example, if in a total orbital reconstruction, the only intact area is medial part of the infraorbital rim, this point could be the reference point and reconstruction will obey the sequence of Inside-outside.

The other concept is confirming points which indicate the areas that should be checked out - and of course should be reduced and fixed- in order to become sure that the tridimensional orientation of the facial complex is precise. We have three confirming points for the midface: ZS suture (zygomatico-sphenoid suture), Root of zygomatic arch and zygomatic buttress.

Case Report I

A 19-years-old male patient has been referred to Maxillofacial surgery ward of Sina hospital with diagnosis of panfacial fracture due to motor vehicle accident (MVA) 1.5 months before referral.

The clinical findings were as follows:

1. Loss of right mandibular premolars, 2. Right comminuted ZMC fracture, 3.Right comminuted Mandibular body fracture, 4. Right hemi Lefort I fracture, 5. Right Coronoid fracture, 6. Right ectropion due to severe multiple facial scars, 7. Severe Malocclusion and 8. Paresis of right Facial nerve. 9. Malnutrition as the sequela of the trauma (Fig-1 \&2).

in order to decrease the operation time, because of malnutrition of the patient, treatment plan was planned to be in two phases in a way that treatment of ZMC fracture was postponed to second phase.

In the first phase, via the vestibular approaches in maxilla and mandible and also through old facial scar, we had an access to the fractures in Maxilla and Mandible. The arch bars were placed and fixed over the dentition in both jaws and also two IMF screws was placed in right molar area of mandible.

After osteotomy and removal of callus from the fracture segments in mandible and maxilla and mobilization of segments, intermaxillary fixation was done. For fixation we started with the simplest fracture area; i.e. Hemi Le fort I fracture with the aid of a miniplate placed at pyriform rim. After that, a mandibular plate was used at mandibular body for fixation. The gap in the fracture site of mandibular body was filled with coronoid process, which had been osteotomized, and callus and grafts were fixed with a miniplate.

At the end scar revision was done.

At the following photos, you can see the Pre- and Post-operative CT scans and clinical views (4months thereafter) of the patient (Fig-3\&4).

The second surgical phase was done 4 months after the first phase (5.5 month after trauma):

In this phase, the approaches were hemicoronal, transconjunctival (retroseptal) and maxillary vestibular. In this patient the intact area was upper face so the most suitable area to start fixation or the Reference point was frontozygomatic suture (ZFS).

On the other hand, ZFS has to be reduced and fixed based on the Confirming point adjacent to it: zygomaticosphenoid suture (ZS). In other words, the position of ZS suture would confirm the accuracy of reduction at ZFS and its tridimensional orientation. In this case, due to defect at ZS suture, we didn't have this confirming point and not only we had to rely on other Confirming points for exact orientation of ZMC, but also we had to reconstruct the Lateral Orbital wall with titanium mesh to prevent postoperative enophthalmos (Fig-5).

After fixation of zygomaticofrontal suture, horizontal pillars should be reconstructed. The first horizontal pillar was zygomatic arch that was fixed with the aid of a microplate in order to prevent postoperative pseudozygomatic arch bowing. Root of zygomatic arch in cases, which fracture line is located at posterior part of arch, has to be checked out as the second Confirming point (Fig-6). 
The second horizontal pillar was Inferior Orbital rim. In this case, medial orbital wall was intact so internal orbital reconstruction was done only to cover the defect at Orbital floor with titanium mesh and temporal fascia placed over it, paying attention to Orbital axis and the edge of defect (Fig-7).

Next the large defect from infraorbital rim up to maxillary alveolar process in anterior wall of maxillary sinus was covered with another Titanium mesh.

The third confirming point is Zygomatic buttress that was fixed using a mini plate. After that ORIF at pyriform rim was repeated.

Finally, Zygomaticus Major muscle was suspended with 2-0 Nylon suture.

The following photos show the comparative CT scan (Pre- and Post-operative) (Fig-8).

Nine months after the second surgery, the third surgery was scheduled. The problem list was: 1. Right Zygomatic arch bowing (was diagnosed 2 months post-op), 2. Depression at right Temporal fossa and 3. Mild right enophthalmos (Fig-9).

The approaches were hemicoronal and transconjunctival (retroseptal) approaches.

Intraoperatively we found out fracture of microplate over the zygomatic arch and separation of bone segments; that's why zygomatic arch bowing was manifested 2 months postoperatively.

The treatment plan was as follow: at first the plates at zygomatic arch and zygomaticofrontal suture were removed and the segments of Zygomatic arch was re-fixed was with two miniplates. Internal Orbital reconstruction was adjusted by releasing of soft tissue from titanium mesh and adding a double-layered medpore. Finally, temporal depression was augmented with two sheets of medpore (Fig-10).

You can see the final results in the following photo (Fig-11).

Case Report II

A 25-years-old male patient was referred to Maxillofacial surgery ward of Sina hospital with diagnosis of panfacial fracture due to motor vehicle accident (MVA) one month before referral.

The clinical findings were as follows: 1 . No light perception at right eye, 2. Scar over the dorsum of the nose, 3. Telecanthus with $40 \mathrm{~mm}$ intercanthal distance, 4. Saddle nose and 5. Mild enophthalmus (Fig-12).

In summery we had: right Lefort II fracture, left Lefort I fracture and Lefort III fracture, undisplaced palatal fracture, nasoorbitoethmoidal (NOE) type II fracture, frontal bone (glabella) fracture and comminuted nasal bone fracture (Fig-13).

For this patient (just like the $1^{\text {st }}$. case) we preferred to use submental intubation and the approaches were bicoronal approach, vestibular approach for maxilla, bilateral transconjunctival approach (retroseptal + Lat. Canthotomy) and H- type approach over the nose including old scar on the nose.

The concept of "Top to bottom" and "Inside to outside" was selected so initially upper midface including glabella \& dorsum of the nose were reconstructed. For this aim two nasal bones and frontal processes of maxillary bone were fixed to frontal bone using microplates then other segments of nasal bones were fixed with another microplates. Then with the aid of a $0.4 \mathrm{~mm}$ SS wire (creating bur holes at the Med. orbital walls and passing the wire like a mattress suture), we constricted and elevated the NOE complex in order to deal with telecanthus. Then we grasped the Med. canthal tendon at each side with the 2-0 Nylon suture and fixed it to the contralateral plate in order to have a support for the ligaments. You can see all these procedures in the following photos with indicating the wire and the sutures with purple flashmarks. Final intercanthal distance was decreased to $35 \mathrm{~mm}$. In next step Frontozygomatic suture and Lateral Orbital wall were fixed, paying attention to Zygomaticosphenoid sutures as a confirming point and after that right and left inferior orbital rims were fixed (Fig-14). 
Since bilateral zygomatic arches were relatively not displaced, the next step was fixation of left Zygomatic buttress and right Pyriform rim (Fig-15).

Consequently, a monocortical calvarial graft was harvested in order to elevate the nasal dorsum. This graft was then covered with temporal fascia \& was fixed on its place using suture passing through upper lateral cartilage and over the graft (Fig-16).

In the following photos you can compare the Pre- and Post-operative CT scans (Fig-17).

\section{Case Report III}

A 37-years-old male patient was referred to Maxillofacial surgery ward of Sina hospital with the diagnosis of Panfacial Fx. due to motor vehicle accident (MVA) 5 days before referral.

According to clinicoradiographic findings, the pattern of the fracture was as follows: Symphyseal Fx, Lefort I Fx, Right Lefort II Fx, Left Lefort III Fx. Maxillary and Mandibular dentoalveolar Fx.s, and Frontal bone Fx.

Some degree of pure blow out fracture was seen \& sagittal cut of CT scan revealed a spur-shaped bone fragment resulting restriction of ocular muscle movement (Fig-18).

First mandible has been reduced and fixed as a Reference point. In other words, after reduction of dentoalveolar fractures in both jaws with the aid of Arch bar and IMF, the fracture line at symphysis was exposed via Vestibular approach and was reduced and fixed with 2 miniplates. At upper- and midface we preferred to have the concept "Up to bottom". Via Bicoronal approach, frontal B., nasofrontal suture and ZFS were exposed, reduced and fixed. Then with a wire we stablished a bone fragment at sup. Orbital rim. Next step was doing ORIF over pyriform rim \& zygomatic buttress via vestibular approach.

Finally, internal orbital reconstruction of left eye was done through transconjunctival approach and a sheet of Porex was inserted in orbital floor to cover the defect. The postoperative photographies were obtained 2 months after operation.

At the following photos you can see the intraoperative and postoperative photos of the patient and compare the Pre- and Post-operative CT scans (Fig-19 to 21).

Discussion

There are different etiological factors for panfacial fractures according to geographical, socioeconomical and cultural characteristics of the society $(5,6)$. In our society motor vehicle accidents \& motor cycle accidents are the most common causes.

The main goal in treatment of panfacial fractures is repositioning of fracture segments and reconstruction of previous facial contour and more importantly occlusion(7).

There are various concepts regarding sequencing at reduction \& fixation of fracture segments in managing a panfacial Fx. (1). Furthermore there are different approaches for exposure and fixation based on experience of the surgeon(8). Some surgeons considered reconstruction of occlusion as the first step in treatment and begin with mandible. Therefor they pay attention to vertical height of the face by restoring the situation of maxillomandibular unit and then the rest of the face.

In fact they follow the sequence of "Bottom to top" (9).

On the other hand some surgeons addresses establishing facial width to reconstruct facial projection and consequently establishing the facial height, following the concept of "Inside to outside" (2).

An important point in reduction of maxillofacial skeleton is paying attention to the horizontal and vertical pillars of the face that make a strong framework for face and a good pathway to transmit the mastication forces (10). These pillars which should be reduced properly and stabilized firmly (1). No matter which 
concept has been chosen, the exact three-dimensional orientation of face has to be checked out by confirming points .

Conclusion

In this study, we have tried to emphasize the importance of "Reference point and Confirming points" in term of reduction and fixation of fracture segments besides the "concepts of sequencing" through 3 cases. Although choosing the most conservative approaches to expose the fracture segments has always been the priority of surgeons, management of panfacial fracture needs more aggressive approaches.

Author Contribution:

Mehrnoush Momeni Roochi: study concept, study design, manuscript editing and review

Narges Hajiani: manuscript preparation, editing and review

Sayna Nezaminia: manuscript preparation.

Reverences

1. Changule G, Mahindra U, Kadam V, Harde K. MANAGEMENT OF PANFACIAL TRAUMA: A SURGICAL QUANDARY FOR MAXILLOFACIAL SURGEONS. Int J Sci Res. 2020;8(12).

2. Mundinger GS, Gruss JS, Hopper RA. Management of Panfacial Fractures. Facial Trauma Surgery: Elsevier; 2020. p. 248-60.

3. Jin HB, Chung JH, Kim KS, Kim SH, Choe J, Yang JY. Le Fort I osteotomy as treatment for traumatic class III malocclusion caused by Le Fort III fracture: A case report. Arch Aesthetic Plast Surg. 2019;25(1):326 .

4. Abouchadi A, Taoufik H, Nacir O, Arrob A. Pan-Facial Fractures: A Retrospective Study and Review of Literature. Open Journal of Stomatology. 2018;8(4):110-9.

5. Mobark MM, Hussein HM, Hassanen KA, Rizk AG. Pattern and Treatment Outcome of Panfacial Fractures in Sohag University Hospital. SVU-IJVS. 2018;2(1).

6. Abdelrahman TEF, Abdelmaaboud A, Hamody A. Challenge and management outcome of panfacial fractures in Sohag University hospital, Egypt. Int Surg J. 2017;5(1):126-31.

7. Vasudev S, Vakade C, Bansal A, Paramesh R. PAN-FACIAL FRACTURES-REVIEW OF LITERATURE AND CASE SERIES. Annals of Dental Specialty Vol. 2016;4(3):86.

8. Hihara M, Yagura T, Takegawa M, Kakudo N, Morimoto N, Kusumoto K. A novel fixation method for panfacial fracture using an Ilizarov-type external fixator. Trauma case rep. 2019;22:100214.

9. Ali K, Lettieri SC, editors. Management of panfacial fracture. Seminars in plastic surgery; 2017: Thieme Medical Publishers.

10. Yun S, Na Y. Panfacial bone fracture: cephalic to caudal. Arch of craniofac surg. 2018;19(1):1.

\section{Hosted file}

fig.docx available at https://authorea.com/users/330642/articles/457423-reference-point-andconfirming-points-two-important-guides-in-treatment-of-panfacial-fx-3-case-reports 